\title{
Prevalence and Clinical Features of Autosomal Dominant and Recessive TMC1-Associated Hearing Loss.
}

Shin-ya Nishio

Shinshu University School of Medicine

Shin-ichi Usami ( $\square$ usami@shinshu-u.ac.jp)

Shinshu Daigaku https://orcid.org/0000-0002-5068-6122

\section{Research Article}

Keywords: TMC1, Next-generation sequencing, Hearing loss, Autosomal dominant, Autosomal recessive, Founder mutation

Posted Date: June 4th, 2021

DOI: https://doi.org/10.21203/rs.3.rs-581891/v1

License: () (7) This work is licensed under a Creative Commons Attribution 4.0 International License. Read Full License

Version of Record: A version of this preprint was published at Human Genetics on September 14th, 2021. See the published version at https://doi.org/10.1007/s00439-021-02364-2. 


\section{Abstract}

TMC1 is a causative gene for both autosomal dominant non-syndromic hearing loss (DFNA36) and autosomal recessive non-syndromic hearing loss (DFNB7/11). To date, 125 pathogenic variants in $T M C 1$ have been reported. Most of the $T M C 1$ variants are responsible for autosomal recessive hearing loss, with only 7 variants reported as causative for DFNA36. Here we reported the prevalence of $T M C 1$-associated hearing loss in a large non-syndromic hearing loss cohort of about 12,000 subjects. As a result, we identified 26 probands with TMC1-associated hearing loss and the estimated prevalence of TMC1associated hearing loss in the Japanese hearing loss cohort to be $0.18 \%$ among all patients. Among the 26 probands with $T M C 1$-associated hearing loss, 15 cases were identified from autosomal dominant hearing loss families. By using the audiometric data from the probands, family members and previously reported cases, we evaluated the hearing deterioration speed for DFNA36 patients. In addition, we performed haplotype analysis for 11 unrelated autosomal dominant hearing loss families carrying the same variant TMC1: NM_138691:c.1627G >A:p.D543N. The results clearly indicated that the same haplotype was present despite of families being unrelated, supporting the contention that this variant occurred by founder mutation.

\section{Introduction}

Hearing loss is one of the most common sensory disorders and, currently, approximately 120 genes have been reported as causative for non-syndromic hearing loss (The Hereditary Hearing Loss Homepage). TMC1 is a causative gene for both autosomal dominant non-syndromic hearing loss (ADNSHL) and autosomal recessive non-syndromic hearing loss (ARNSHL) as first reported by Kurima et al (2002). The encoding protein transmembrane channel-like protein 1 is highly expressed in the tips of stereocilia and plays a crucial role in mechano-electro-transduction (Liu et al., 2020).

TMC1 variants are a relatively common genetic cause of non-syndromic hearing loss, and accounts for 3.4\% of Pakistani ARNSHL (Kitajiri, McNamara, et al., 2007), 4.3 to $8.1 \%$ of Turkish (Kalay et al., 2005; Sirmaci et al., 2009), 5.9\% of Tunisian (Tlili et al., 2008), 4.2\% of European (Schrauwen et al., 2013), and 2.3\% of American (Sloan-Heggen et al., 2016) hearing loss patients. Most cases of TMC1-associated hearing loss are identified from autosomal recessive inherited hearing loss, and only limited cases are identified as autosomal dominant. The clinical phenotypes of TMC1-associated hearing loss differ according to the inheritance mode. TMC1-associated ARSNHL cases show congenital severe-to-profound hearing loss, whereas ADSNHL cases show late-onset progressive hearing loss with predominant deterioration in the higher frequencies. To date, 125 pathogenic variants in TMC1 have been reported (HGMD Professional). Among the 125 pathogenic variants, only 8 variants were reported as causative for ADNSHL (DFNA36). The TMC1 gene variants associated with ADNSHL are p.I266T (Sloan-Heggen 2016), p.S320R (Hassan et al., 2015), p.Y381N (Likar et al., 2018), p.G417R (Yang et al., 2010), p.M418K (Zho et al., 2014; Wang et al., 2018), p.D543N (Moteki et al., 2016), p.D572N (Kurima et al., 2002; Wang et al., 2018; Ramzan et al., 2019), and p.D572H (Kitajiri et al., 2007). However, there is some conflict regarding the pathogenicity of the p.D572H variants (Azaiez et al., 2018). In addition, the p.I266T variant and p.Y381N variant were also reported as causative for TMC1-associated ARSNHL (Wang et al., 2018; Sommen et al., 2016). So, only five variants identified from 8 families are reliably known to be the genetic cause of TMC1-associated ADNSHL. Based on this limited number of cases, the overall picture regarding the clinical phenotypes of TMC1-associated ADNSHL remains unclear.

Recently, autosomal dominant TMC1-associated hearing loss has received special attention as a candidate for gene therapy. A mouse model of TMC1-related hearing loss (Beethoven mice), which are generated by ENU mutagenesis, showed autosomal dominant inherited progressive hearing loss (Vreugde et al., 2002). This mouse model carries the Tmc1:c. 1235 T > A:p.M412K variant and, subsequent to this report, ADNSHL patients with an orthologous TMC1 variant (TMC1 c.1253T > A:p.M418K) were reported (Zhao et al., 2014). As the Beethoven mice showed a similar phenotype (progressive hearing loss with predominant deterioration in the higher frequencies) to human patients and carried the orthologous mutation identified in human ADNSHL patients, this mouse model is widely used for translational research for gene therapy (Askew et al., 2015; Shibata et al., 2016; Yoshimura et al., 2018; Gao et al., 2018; NistLund et al., 2019; György et al., 2019; Wu et al., 2021). However, prior to the clinical application of gene therapies, the detailed phenotypes and prevalence information are essential.

In this study, we sought to (1) elucidate the prevalence of HL caused by TMC1 variants in a large cohort of non-syndromic hearing loss patients, (2) analyze the rate of $\mathrm{HL}$ deterioration in TMC1-associated ADNSHL patients, and (3) carry out haplotype analysis of the TMC1: NM_138691:c.1627G > A:p.D543N variant identified from 11 unrelated ADNSHL families to confirm whether the mutation occurred by founder mutation or in a mutational hotspot.

\section{Methods}

\section{Subjects}

We performed target re-sequencing analysis for 12,139 Japanese non-syndromic sensorineural hearing loss patients and controls (2,462 autosomal dominant or mitochondrial inheritance cases, 6,912 autosomal recessive inheritance or sporadic cases, 2,220 unknown family history cases, 212 cases with unilateral hearing loss, and 333 control subjects) from 90 otorhinolaryngology departments nationwide enrolled in this study. In addition, we also analyzed for 187 cochlear implant patients or electric acoustic stimulation patients enrolled from 10 cochlear implantation centers listed below: Antwerp University Hospital, Belgium (Prof. Paul Van de Heyning); Hospital Universitario La Paz, Spain (Prof. Javier Gavilán); Klinikum der Universität München, German (Prof. Joachim Müller); Karolinska University Hospital, Sweden (Prof. Eva Karltorp); Institute of Physiology and Pathology of Hearing, Poland (Dr. Henryk Skarzynski and Dr. Piotr Skarzynski); King Abdulaziz University Hospital, Saudi Arabia (Prof. Abdulrahman Hagr), ENT Super Speciality Institute and Research Center, India (Dr. Manikoth Manoj); University of Western Australia, Australia (Prof. Gunesh Rajan); Kansas University, USA (Prof. Hinrich Staecker); and Allende Sanatorio, Argentina (Dr. Mario Zernotti).

Informed written consent was obtained from all subjects (or guardians in the case of minors) prior to participation. This study was approved by the Shinshu University Ethics Committee (Approval number: 576) and the respective ethics committees of all other participating institutions. 
Next-generation sequencing was performed for the 63 genes reported to cause nonsyndromic hearing loss described in a previous report (Nishio et al., 2015). In brief, amplicon libraries were prepared by using the lon AmpliSeq Custom Panel, with the lon AmpliSeq Library Kit 2.0 and the lon Xpres Barcode Adapter 196 Kit (Life Technologies) according to the manufacturer's instructions. After amplicon library preparation, equal amounts of libraries of forty-five patients were pooled for one sequence reaction and next-generation sequencing was performed by lon Proton system with an lon P1 chip or lon S5 system with an lon 540 chip according to the manufacturer's instructions. The sequence data were aligned to the human reference genome sequence (build GRCh37/hg19) by the Torrent Mapping Alignment Program (TMAP) and, subsequently, DNA variants were piled up with the Torrent Variant Caller plug-in software including in the Torrent Suit (Life Technologies).

The effects of the variants were analyzed by using ANNOVAR software (Wang et al., 2010). The missense, nonsense, insertion/deletion, and splicing variants were selected among the identified variants. Variants were further selected as $<1 \%$ of several control database including the 1,000 genome database (http://www.1000genomes.org/), the 6,500 exome variants (http://evs.gs.washington.edu/EVS/), The Genome Aggregation Database (https://gnomad.broadinstitute.org), the human genetic variation database (dataset for 1,208 Japanese exome variants) (http://www.genome.med.kyotiu.ac.jp/SnpDB/index.html), the 8,300 Japanese genome variation database (https://jmorp.megabank.tohoku.ac.jp/202102/) and the 333 in-house Japanese normal hearing controls. All filtering procedures were performed by using original database software described previously (Nishio and Usami 2017). The pathogenicity of the identified variants was evaluated in accordance with the American College of Medical Genetics (ACMG) standards and guidelines (Richards et al., 2015) with the ClinGen hearing loss clinical domain working group expert specification (Oza et al., 2018). We performed Sanger sequencing analysis to validate the identified variants by using PCR and exon specific custom primers according to the manufacturer's instructions. All primers were designed using the web version Primer 3 plus software (http://www.bioinformatics.nl/cgi-bin/primer3plus/primer3plus.cgi).

\section{Haplotype Analysis}

The haplotype pattern within the 3Mbp region surrounding the frequent Japanese variation TMC1: NM_138691:c.1627G>A identified in this study was analyzed using a set of 47 single nucleotide polymorphisms (SNPs) (21 sites for upstream and 26 sites for downstream). For this analysis, we selected 15 individuals (including 11 affected and 4 un-affected family members) from 5 families. Haplotype analysis was performed by Sanger sequencing. The mutation-linked haplotype was determined by family member segregation analysis with multiple family member samples, and compared among unrelated families with the same mutations.

\section{Results}

\section{Identified variants, prevalence, and the clinical features of TMC1-associated hearing loss}

As a result of the large cohort of next-generation sequencing analysis, we identified 26 probands with TMC1-associated hearing loss (Table 1 and Supplemental Fig. 1). Among the 26 probands, 15 were identified from ADNSHL or maternally inherited cases, whereas 11 were identified from ARNSHL or sporadic cases. No other candidate pathogenic variants in the other 62 deafness genes were identified in these 26 probands. When we restricted analysis to Japanese bilateral non-syndromic hearing loss patients, the prevalence of TMC1-associated hearing loss was $0.17 \%(20 / 11,594)$ for all patients, $0.61 \%$ $(15 / 2,462)$ for ADNSHL and $0.07 \%(5 / 6,912)$ for ARNSHL or sporadic hearing loss cases. 
Table 1

TMC1-associated hearing loss cases identified in this study.

\begin{tabular}{|c|c|c|c|c|c|c|c|c|c|c|}
\hline & & Variant 1 & & Variant 2 & & & & & & \\
\hline ID & Inheritance & Base change & AA change & Base change & AA change & ethnicity & $\begin{array}{l}\text { Type } \\
\text { of } \\
\mathrm{HL}\end{array}$ & $\begin{array}{l}\text { Severity } \\
\text { of } \mathrm{HL}\end{array}$ & Progression & Tinı \\
\hline 04886 & $A D$ & c. $1627 \mathrm{G}>\mathrm{A}$ & p.D543N & & & Japanese & Flat & Profound & Yes & Yes \\
\hline 04091 & $A D$ & c. $1627 \mathrm{G}>\mathrm{A}$ & p.D543N & & & Japanese & Flat & Profound & Yes & Yes \\
\hline 05030 & $A D$ & c. $1627 \mathrm{G}>\mathrm{A}$ & p.D543N & & & Japanese & Flat & Moderate & Yes & Yes \\
\hline HL2672 & $A D$ & c. $1627 \mathrm{G}>\mathrm{A}$ & p.D543N & & & Japanese & Flat & Profound & Yes & Yes \\
\hline 00487 & $A D$ & c. $1627 \mathrm{G}>\mathrm{A}$ & p.D543N & & & Japanese & NA & NA & Yes & NA \\
\hline HL6536 & $A D$ & c. $1627 \mathrm{G}>\mathrm{A}$ & p.D543N & & & Japanese & $\begin{array}{l}\text { High } \\
\text { Freq }\end{array}$ & Severe & Yes & NA \\
\hline HL9117 & $A D$ & c. $1627 \mathrm{G}>\mathrm{A}$ & p.D543N & & & Japanese & $\begin{array}{l}\text { High } \\
\text { Freq }\end{array}$ & Moderate & Yes & No \\
\hline HL9205 & $A D$ & c. $1627 \mathrm{G}>\mathrm{A}$ & p.D543N & & & Japanese & NA & Profound & Yes & NA \\
\hline HL9597 & $A D$ & c. $1627 \mathrm{G}>\mathrm{A}$ & p.D543N & & & Japanese & $\begin{array}{l}\text { High } \\
\text { Freq }\end{array}$ & Severe & Yes & Yes \\
\hline HL4994 & $A D$ & c. $1627 \mathrm{G}>\mathrm{A}$ & p.D543N & & & Japanese & NA & NA & NA & NA \\
\hline HL6717 & $A D$ & c. $1627 \mathrm{G}>\mathrm{A}$ & p.D543N & & & Japanese & NA & NA & NA & NA \\
\hline HL3819 & $A D$ & c. $1714 \mathrm{G}>\mathrm{A}$ & p.D572N & & & Japanese & $\begin{array}{l}\text { High } \\
\text { Freq }\end{array}$ & Moderate & NA & NA \\
\hline HL4498 & $A D$ & c. $1714 \mathrm{G}>\mathrm{A}$ & p.D572N & & & Japanese & NA & NA & NA & NA \\
\hline HL8588 & $A D$ & c. $1714 \mathrm{G}>\mathrm{A}$ & p.D572N & & & Japanese & NA & NA & NA & NA \\
\hline HL7492 & $A D$ & c. $1714 \mathrm{G}>\mathrm{A}$ & p.D572N & & & Japanese & NA & NA & NA & NA \\
\hline HL3123 & Sporadic & c. $100 \mathrm{C}>\mathrm{T}$ & p.R34X & c. $884+1 G>A$ & splicing & Japanese & Flat & Profound & No & No \\
\hline HL3604 & Sporadic & c. $210 \mathrm{delG}$ & p.R71Gfs*5 & c. $1592 A>T$ & p.D531V & Japanese & Flat & Profound & No & NA \\
\hline HL7927 & Sporadic & c. $741+1 \_+4$ del & splicing & c. $1333 \mathrm{C}>\mathrm{T}$ & p.R445C & Japanese & Flat & Severe & NA & NA \\
\hline HL4017 & Sporadic & c. $1165 \mathrm{C}>\mathrm{T}$ & p.R389X & c. $1165 \mathrm{C}>\mathrm{T}$ & p.R389X & Japanese & Flat & Profound & No & No \\
\hline HL8573 & AR & c.2047_2048del & p.H683Rfs*169 & c.2047_2048del & p.H683Rfs*169 & Japanese & Flat & Profound & NA & NA \\
\hline MED473 & Sporadic & c.247_249del & p.E83del & c.247_249del & p.E83del & German & NA & NA & No & No \\
\hline MED214 & Sporadic & c. $338 \mathrm{~T}>\mathrm{C}$ & p.M113T & c. $1534 \mathrm{C}>\mathrm{T}$ & p.R512X & Swedes & $\begin{array}{l}\text { High } \\
\text { Freq }\end{array}$ & Severe & NA & NA \\
\hline MED131 & Sporadic & c. $674 \mathrm{C}>\mathrm{T}$ & p.P225L & c. $1333 \mathrm{C}>\mathrm{T}$ & p.R445C & Polish & Flat & Profound & No & No \\
\hline MED097 & Sporadic & c.1235delT & p.M413Cfs*4 & c. $1764 \mathrm{G}>\mathrm{A}$ & p.W588X & Polish & $\begin{array}{l}\text { High } \\
\text { Freq }\end{array}$ & Profound & No & No \\
\hline MED138 & AR & c. $1764 \mathrm{G}>\mathrm{A}$ & p.W588X & c. $1764 \mathrm{G}>\mathrm{A}$ & p.W588X & Polish & $\begin{array}{l}\text { High } \\
\text { Freq }\end{array}$ & Profound & No & No \\
\hline MED430 & Sporadic & c.2176_2177del & p.A726Efs*126 & c.2176_2177del & p.A726Efs*126 & Indian & Flat & Profound & No & No \\
\hline
\end{tabular}

* All variants are indicated on NM_138691.

AA: amino acid, AD: autosomal dominant, AR: autosomal recessive, NA: not available

The variants identified in this study are summarized in Table 2 . In this study, we identified 17 candidate TMC1 variants, 7 of which were novel variants and 10 were previously reported. Based on ACMG guidelines and ClinGen HLCDWG expert specifications, 5 were classified as "pathogenic" variants and 2 were classified as of "uncertain significance". Interestingly, TMC1:c.1627G > A:p.D543N variants and TMC1:c.1714G > A:p.D572N variants were identified from 11 and 4 unrelated families with ADNSHL, respectively. Both variants were only identified from ADNSHL patients and were not identified from 6,912 autosomal recessive inheritance or sporadic cases, or 2,220 unknown family history cases. In addition, these variants were not identified in the gnomAD database or 8.3KJPN (Japanese 8,380 genomic variant database). Both of above results strongly supported the pathogenicity of these variants as causative for TMC1associated ADNSHL. 
Table 2

TMC1 variants identified in this study.

\begin{tabular}{|c|c|c|c|c|c|c|c|c|c|c|c|c|}
\hline Base change & AA change & Inheritance & SIFT & PP2 & MutTaster & REVEL & CADD & 8.3KJPN & gnomAD & AD_MAF & AR_MAF & Cling \\
\hline c. $100 \mathrm{C}>\mathrm{T}$ & p.R34X & AR & - & - & A & - & 36 & 0 & 0.000056 & 0 & 0.00018 & \\
\hline c. $210 \mathrm{delG}$ & p.R71Gfs*5 & $\mathrm{AR}$ & - & - & - & - & - & 0.0001 & 0 & 0 & 0.00018 & Patho \\
\hline c.247_249del & p.E83del & AR & - & - & - & - & - & 0 & 0 & 0 & 0.00036 & \\
\hline c.338T > C & p.M113T & AR & D & $\mathrm{P}$ & $\mathrm{D}$ & 0.263 & 24.8 & 0 & 0.000004 & 0 & 0.00018 & VUS \\
\hline c. $674 \mathrm{C}>\mathrm{T}$ & p.P225L & $A R$ & $\mathrm{~T}$ & $\mathrm{D}$ & D & 0.4 & 27.2 & 0 & 0.000044 & 0 & 0.00018 & \\
\hline c.741+1_+4del & $\mathrm{spl}$ & $\mathrm{AR}$ & - & - & - & - & - & 0 & 0 & 0 & 0.00036 & Patho \\
\hline c. $884+1 G>A$ & spl. & AR & - & - & D & - & 27.2 & 0 & 0.000012 & 0 & 0.00012 & \\
\hline c. $1165 \mathrm{C}>\mathrm{T}$ & p.R389X & $\mathrm{AR}$ & - & - & $A$ & - & 38 & 0 & 0.000068 & 0 & 0.00054 & \\
\hline c.1235delT & p.M413Cfs*4 & $A R$ & - & - & - & - & - & 0 & 0 & 0 & 0.00018 & Patho \\
\hline c. $1333 \mathrm{C}>\mathrm{T}$ & p.R445C & $A R$ & $\mathrm{D}$ & $\mathrm{D}$ & D & 0.662 & 35 & 0 & 0.000072 & 0 & 0.00036 & \\
\hline c. $1534 \mathrm{C}>\mathrm{T}$ & p.R512X & $\mathrm{AR}$ & - & - & A & - & 42 & 0 & 0.0003 & 0 & 0.00018 & \\
\hline c. $1592 A>T$ & p.D531V & AR & $\mathrm{D}$ & D & D & 0.861 & 25.7 & 0 & 0 & 0 & 0.00018 & VUS \\
\hline c. $1627 \mathrm{G}>\mathrm{A}$ & p.D543N & $A D$ & $\mathrm{D}$ & $\mathrm{D}$ & D & 0.472 & 32 & 0 & 0 & 0.0082 & 0 & \\
\hline c. $1714 \mathrm{G}>\mathrm{A}$ & p.D572N & $A D$ & $\mathrm{~T}$ & $\mathrm{D}$ & D & 0.465 & 29.7 & 0 & 0 & 0.0045 & 0 & \\
\hline c. $1764 \mathrm{G}>\mathrm{A}$ & p.W588X & $\mathrm{AR}$ & - & - & A & - & 42 & 0 & 0.000012 & 0 & 0.00054 & \\
\hline c.2047_2048del & p.H683Rfs*169 & $\mathrm{AR}$ & - & - & - & - & - & 0 & 0 & 0 & 0.00036 & Patho \\
\hline c.2176_2177del & p.A726Efs ${ }^{\star} 126$ & AR & - & - & - & - & - & 0 & 0 & 0 & 0.00036 & Patho \\
\hline
\end{tabular}

AA: amino acid, AD: autosomal dominant, AR: autosomal recessive, PP2: PolyPhen2, MutTaster: Mutation Taster, AD_MAF: Minor allele frequency in ADNSHL allele frequency in ARNSHL cases,

In terms of clinical features, TMC1-associated ARNSHL patients showed congenital onset severe-to-profound hearing loss, whereas the TMC1-associated ADNSHL patients showed late-onset progressive hearing loss (Table 1). The severity of hearing loss in ADNSHL patients varied from moderate to severe hearing loss depending on patient age. In addition, 3 family members of family \#04886 who carried TMC1:c.1627G >A:p.D543N variants showed normal hearing (Supplemental Fig. 1). Most of the ADNSHL cases complained of the progression of hearing loss and tinnitus; however, only two patients suffered episodes of vertigo.

\section{Hearing deterioration speed for TMC1-associated ADNSHL}

Most of the TMC1-associated ARSNHL patients showed congenital severe-to-profound hearing loss. On the other hand, TMC1-associated ADNSHL patients showed late-onset progressive hearing loss (Table 1). To elucidate the hearing deterioration speed for TMC1-associated ADNSHL, we performed regression analysis of age and hearing thresholds of 250,500, 1000, 2000, 4000 and $8000 \mathrm{~Hz}$ (Fig. 1). For this analysis, we used the hearing thresholds for 11 probands and 12 family members identified in this study. In addition, we also included the 34 hearing thresholds data for 24 affected individuals with TMC1-associated ADNSHL from previous reports (Kurima et al., 2002; Yang et al., 2010; Zhao et al., 2014; Wang et al., 2018). As shown in Fig. 1, the hearing levels in the higher frequencies deteriorate more rapidly than in the lower frequencies. The estimated hearing deterioration speed in terms of pure-tone average (average of $500 \mathrm{~Hz}$ $1000 \mathrm{~Hz}, 2000 \mathrm{~Hz}$ and $4000 \mathrm{~Hz}$ ) was $1.0 \mathrm{~dB}$ per year.

\section{Haplotype Analysis}

Interestingly, 11 unrelated Japanese ADNSHL families carried the same variant (TMC1: NM_138691:c.1627G > A:p.D543N). We, therefore, carried out haplotype analysis to confirm whether this mutation occurred by founder mutation or in a mutational hotspot. Figure 2 shows the haplotype patterns for four unrelated families who carried the same TMC1: NM_138691:c.1627G > A variant. As a result, four unrelated families were found to carry the same haplotype in the $1.3 \mathrm{Mbp}$ region surrounding this mutation (the preserved region ranged from $0.7 \mathrm{Mbp}$ upstream to $0.6 \mathrm{Mbp}$ downstream) suggesting that this mutation occurred and spread as founder mutation in Japanese populations.

\section{Discussion}


In this study, we identified 27 probands with TMC1-associated hearing loss (15 were identified from ADNSHL cases, whereas 11 were identified from ARNSHL or sporadic cases). The prevalence of $T M C 1$-associated hearing loss in Japanese hearing loss patients was $0.17 \%$ for all patients, $0.61 \%$ for ADNSHL and $0.07 \%$ for ARNSHL or sporadic hearing loss cases. The prevalence of TMC1-associated hearing loss in other countries was higher than that in our Japanese cohort: 3.4\% in Pakistani ARSNHL patients (Kitajiri et al., 2007), 4.3 to 8.1\% in Turkish (Kalay et al., 2005; Sirmaci et al., 2009), 5.9\% in Tunisian (Tlili et al., 2008), 4.2\% in European (Schrauwen et al., 2013), and 2.3\% in American (Sloan-Heggen et al., 2016) hearing loss patients. These differences may be caused by the carrier frequencies of commonly observed mutations. In most previous studies, TMC1-associated hearing loss was observed more commonly from ARNSHL patients than from ADNSHL patients, and common mutations which may be caused by founder mutation, were involved in these cases. On the other hand, in our Japanese hearing loss cohort, ADNSHL cases were more commonly observed than ARNSHL cases. In addition, all identified variants from Japanese TMC1-associated ARNSHL cases differed among patients and no common mutations were identified.

Similar to previous studies, TMC1-associated ARNSHL patients showed congenital onset severe-to-profound hearing loss, whereas the TMC1-associated ADNSHL patients showed late-onset progressive hearing loss. Indeed, 3 younger agers in family \# 04886 showed normal hearing although they carried the same mutation as the other affected family members (Supplemental Fig. 1), supporting the late-onset of their hearing loss. In addition, we also clarified the hearing deterioration speed for DFNA36 by using the hearing threshold data obtained in this study and previous reports, and revealed the hearing deterioration speed in terms of pure-tone average was $1.0 \mathrm{~dB}$ per year.

Toward the clinical application of gene therapy for hereditary hearing loss, TMC1-associated hearing loss is believed to be a good candidate. The ENU-induced mutagenic mouse model, Beethoven mice, carry the Tmc1:c. 1235 T > A:p.M412K variation, and an orthologous TMC1 variant (TMC1 c.1253T > A:p.M418K) has been reported as causative for DFNA36 (Zhao et al., 2014). Beethoven mice are widely used for translational research for gene therapy and favorable results in the prevention of hearing deterioration in these model mice have been achieved (Askew et al., 2015; Shibata et al., 2016; Yoshimura et al., 2018; Gao et al., 2018; Nist-Lund et al., 2019; György et al., 2019; Wu et al., 2021). In these gene therapy studies, the timing of vector administration is also discussed as most of the studies administrated the gene delivering vector, adeno associated virus (AAV), into the inner ear of neonate mice. However, this timing is equivalent to the developmental stage of the inner ear of the human fetus. Recently, Yoshimura et al (2018) reported gene therapy for 2-to 8-week-old mice and prevented hearing deterioration in these model mice, suggesting the appropriate time-window for gene therapy. In this study, we indicated that the hearing deterioration in DFNA36 patients started from their 1st or 2nd decade (teenagers) and the time-window for gene therapy to prevent hearing deterioration might be wider than previously thought.

In this study, we identified 11 unrelated Japanese ADNSHL families that carried same the variant (TMC1: NM_138691:C.1627G > A:p.D543N). Haplotype analysis of TMC1: NM_138691:c.1627G > A:p.D543N showed the same haplotype among the families with the same mutation. This result suggested that this mutation occurred and spread by founder mutation rather than in a mutational hot spot. This hypothesis was supported by the fact that this mutation was only identified from Japanese hearing loss patients. This is the first report of a founder mutation identified in DFNA36. Based on the higher prevalence (11 patients carried this mutation in our 12,139 hearing loss subjects), this mutation will be a good candidate for the clinical study of gene therapy for DFNA36. On the other hand, the c.1714G > A:p.D572N variant observed in this study may be caused by a mutational hotspot. The p.D572N variant was identified from four Japanese ADNSHL patients in this study, but this variant was also identified from North American, Chinese and Saudi patients (Kurima et al., 2002; Wang et al., 2018; Ramzan et al., 2020; Yuan et al., 2020). The observations of patients from different ethnic background also support the fact that this variant was caused by a mutational hotspot.

In summary, next-generation sequencing analysis successfully identified 11 previously reported mutations and 7 novel variants for TMC1-associated hearing loss. The estimated prevalence of TMC1-associated hearing loss in the Japanese hearing loss cohort was $0.18 \%$ for all patients, $0.65 \%$ for $A D N S H L$ and $0.07 \%$ for ARNSHL or sporadic hearing loss cases. This large cohort study of hearing loss patients provided valuable new insights, particularly with regard to the speed of hearing deterioration in DFNA36 patients. This information will be useful baseline data for future therapeutics including gene therapy.

\section{Declarations}

\section{Acknowledgements}

We thank all participants in the present study and our collaborators for providing samples and clinical information as listed below; Dr. Tsukasa Ito and Dr. Toshinori Kubota (Yamagata University), Dr. Mayuri Okami (Tokai University), Dr. Mariko Kakudo (Hyougo Medical University), Dr. Kenji Ohyama and Dr. Kiyoshi Oda (Touhoku Rousai Hospital), Dr. Tomoko Esaki (Aichi Children Medical Center), Dr. Jun Nakayama (Shiga University), Dr. Yasushi Naito and Dr. Hiroshi Yamazaki (Kobe City Medical Center General Hospital), Dr. Yumi Ohta (Osaka University), Dr. Tetsuya Tono (Miyazaki University), Dr. Ikuyo Miyanohara (Kagoshima University), Dr. Manoj Manikoth (ENT Super Speciality Institute and Research Center, India), Dr. Joachim Müller (Klinikum der Universität München, German), Dr. Eva Karltorp (Karolinska University Hospital, Sweden), and Dr. Henryk Skarzynski, and Dr. Piotr Skarzynski (Institute of Physiology and Pathology of Hearing, Poland).

\section{Funding}

This research was funded by a Health and Labor Sciences Research Grant for Research on Rare and Intractable Diseases and Comprehensive Research on Disability Health and Welfare from the Ministry of Health, Labor and Welfare of Japan (S.U. 20FC1048), and a Grants-in-Aid from Japan Agency for Medical Research and Development (AMED) (S.U.: 17kk0205010h0002, 18ek0109363h0001).

\section{Conflicts of interest}

All authors declare no conflicts of interest in this study. 


\section{References}

1. Askew C, Rochat C, Pan B et al (2015) Tmc gene therapy restores auditory function in deaf mice. Sci Transl Med. 7:295ra108.

2. Azaiez H, Booth KT, Ephraim SS et al (2018) Genomic Landscape and Mutational Signatures of Deafness-Associated Genes. Am J Hum Genet. 103:484497.

3. Gao X, Tao Y, Lamas V et al (2018) Treatment of autosomal dominant hearing loss by in vivo delivery of genome editing agents. Nature. 553:217-221.

4. György B, Nist-Lund C, Pan B et al (2019) Allele-specific gene editing prevents deafness in a model of dominant progressive hearing loss. Nat Med. 25:1123-1130.

5. Hassan MA, Shah AA, Szmida E et al (2015) A TMC1 (transmembrane channel-like 1) mutation (p.S320R) in a Polish family with hearing impairment. J Appl Genet. 56:311-6.

6. Kalay E, Karaguzel A, Caylan R et al (2005) Four novel TMC1 (DFNB7/DFNB11) mutations in Turkish patients with congenital autosomal recessive nonsyndromic hearing loss. Hum Mutat. 26:591.

7. Kitajiri S, Makishima T, Friedman TB, Griffith AJ (2007) A novel mutation at the DFNA36 hearing loss locus reveals a critical function and potential genotype-phenotype correlation for amino acid-572 of TMC1. Clin Genet. 71:148-152.

8. Kitajiri SI, McNamara R, Makishima T et al (2007) Identities, frequencies and origins of TMC1 mutations causing DFNB7/B11 deafness in Pakistan. Clin Genet. 72:546-550.

9. Kurima K, Peters LM, Yang Y et al (2002) Dominant and recessive deafness caused by mutations of a novel gene, $T M C 1$, required for cochlear hair-cell function. Nat Genet. 30:277-284.

10. Likar T, Hasanhodžić M, Teran N et al (2018) Diagnostic outcomes of exome sequencing in patients with syndromic or non-syndromic hearing loss. PLoS One 13:e0188578.

11. Liu S, Wang S, Zou L, Xiong W (2020) Mechanisms in cochlear hair cell mechano-electrical transduction for acquisition of sound frequency and intensity. Cell Mol Life Sci. Online ahead of print.

12. Moteki H, Azaiez H, Booth KT et al (2016) Comprehensive genetic testing with ethnic-specific filtering by allele frequency in a Japanese hearing-loss population. Clin Genet. 89:466-472.

13. Nishio SY, Hayashi Y, Watanabe M, Usami S (2015) Clinical application of a custom AmpliSeq library and ion torrent PGM sequencing to comprehensive mutation screening for deafness genes. Genet Test Mol Biomarkers. 19:209-17.

14. Nishio SY, Usami SI (2017) The Clinical Next-Generation Sequencing Database: A Tool for the Unified Management of Clinical Information and Genetic Variants to Accelerate Variant Pathogenicity Classification. Hum Mutat. 38:252-259.

15. Nist-Lund CA, Pan B, Patterson A et al (2019) Improved TMC1 gene therapy restores hearing and balance in mice with genetic inner ear disorders. Nat Commun. 10:236.

16. Oza AM, DiStefano MT, Hemphill SE et al (2018) Expert specification of the ACMG/AMP variant interpretation guidelines for genetic hearing loss. Hum Mutat. 39:1593-1613.

17. Ramzan K, Al-Owain M, Al-Numair NS et al (2020) Identification of TMC1 as a relatively common cause for nonsyndromic hearing loss in the Saudi population. Am J Med Genet B Neuropsychiatr Genet. 183:172-180.

18. Richards S, Aziz N, Bale S et al (2015) Standards and guidelines for the interpretation of sequence variants: a joint consensus recommendation of the American College of Medical Genetics and Genomics and the Association for Molecular Pathology. Genet Med. 17:405-424.

19. Schrauwen I, Sommen M, Corneveaux JJ et al (2013) A sensitive and specific diagnostic test for hearing loss using a microdroplet PCR-based approach and next generation sequencing. Am J Med Genet A. 161A:145-152.

20. Shibata SB, Ranum PT, Moteki H et al (2016) RNA Interference Prevents Autosomal-Dominant Hearing Loss. Am J Hum Genet. 98:1101-1113.

21. Sirmaci A, Duman D, Oztürkmen-Akay H et al (2009) Mutations in TMC1 contribute significantly to nonsyndromic autosomal recessive sensorineural hearing loss: a report of five novel mutations. Int J Pediatr Otorhinolaryngol. 73:699-705.

22. Sloan-Heggen CM, Bierer AO, Shearer AE et al (2016) Comprehensive genetic testing in the clinical evaluation of 1119 patients with hearing loss. Hum Genet. 135:441-450.

23. Sommen M, Schrauwen I, Vandeweyer G et al (2016) DNA Diagnostics of Hereditary Hearing Loss: A Targeted Resequencing Approach Combined with a Mutation Classification System. Hum Mutat. 37:812-9.

24. Tlili A, Rebeh IB, Aifa-Hmani M et al (2008) TMC1 but not TMC2 is responsible for autosomal recessive nonsyndromic hearing impairment in Tunisian families. Audiol Neurootol. 13:213-218.

25. Vreugde S, Erven A, Kros CJ et al (2002) Beethoven, a mouse model for dominant, progressive hearing loss DFNA36. Nat Genet. 30:257-258.

26. Wang H, Wu K, Guan J et al (2018) Identification of four TMC1 variations in different Chinese families with hereditary hearing loss. Mol Genet Genomic Med. 6:504-513.

27. Wang K, Li M, Hakonarson H (2010) ANNOVAR: functional annotation of genetic variants from high-throughput sequencing data. Nucleic Acids Res. 38:e164.

28. Wu J, Solanes P, Nist-Lund C et al (2021) Single and Dual Vector Gene Therapy with AAV9-PHP.B Rescues Hearing in Tmc1 Mutant Mice. Mol Ther. 29:973988.

29. Yang T, Kahrizi K, Bazazzadeghan N et al (2010) A novel mutation adjacent to the Bth mouse mutation in the TMC1 gene makes this mouse an excellent model of human deafness at the DFNA36 locus. Clin Genet. 77:395-398. 
30. Yoshimura H, Shibata SB, Ranum PT et al (2019) Targeted allele suppression prevents progressive hearing loss in the mature Murine model of Human TMC1 deafness. Mol Ther. 27:681-690.

31. Yuan Y, Li Q, Su Y et al (2020) Comprehensive genetic testing of Chinese SNHL patients and variants interpretation using ACMG guidelines and ethnically matched normal controls. Eur J Hum Genet. 28:231-243.

32. Zhao Y, Wang D, Zong L et al (2014) A novel DFNA36 mutation in TMC1 orthologous to the Beethoven (Bth) mouse associated with autosomal dominant hearing loss in a Chinese family. PLoS One. 9:e97064.

\section{Figures}

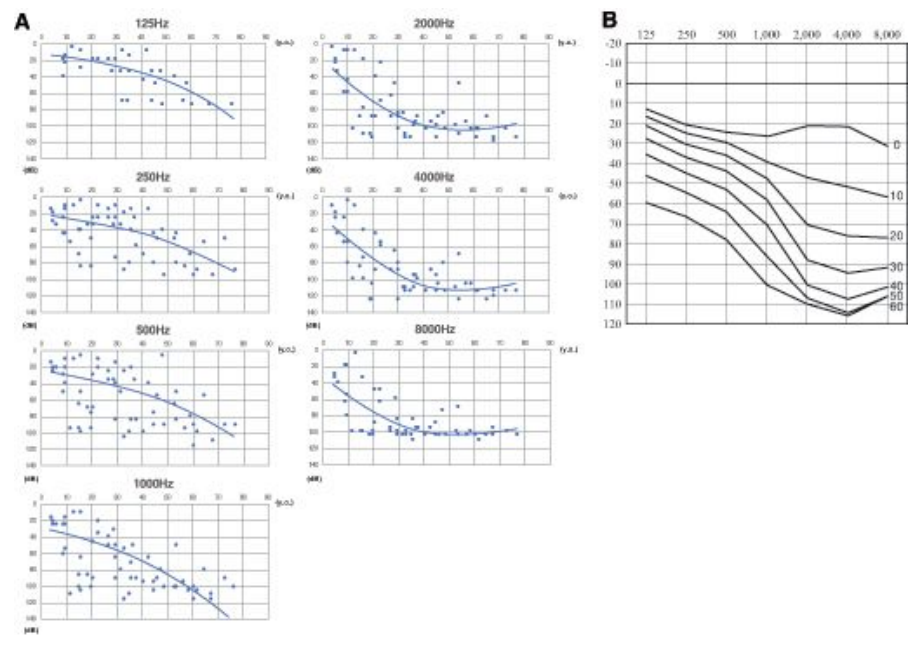

Figure 1

Detailed progression analysis of DFNA36 patients. A: Hearing thresholds from audiograms (the better ear) of the patients identified in this study and previously reported were plotted for each frequency. B: Estimated age-related typical audiogram (ARTA) demonstrating the progression of hearing loss for DFNA36.

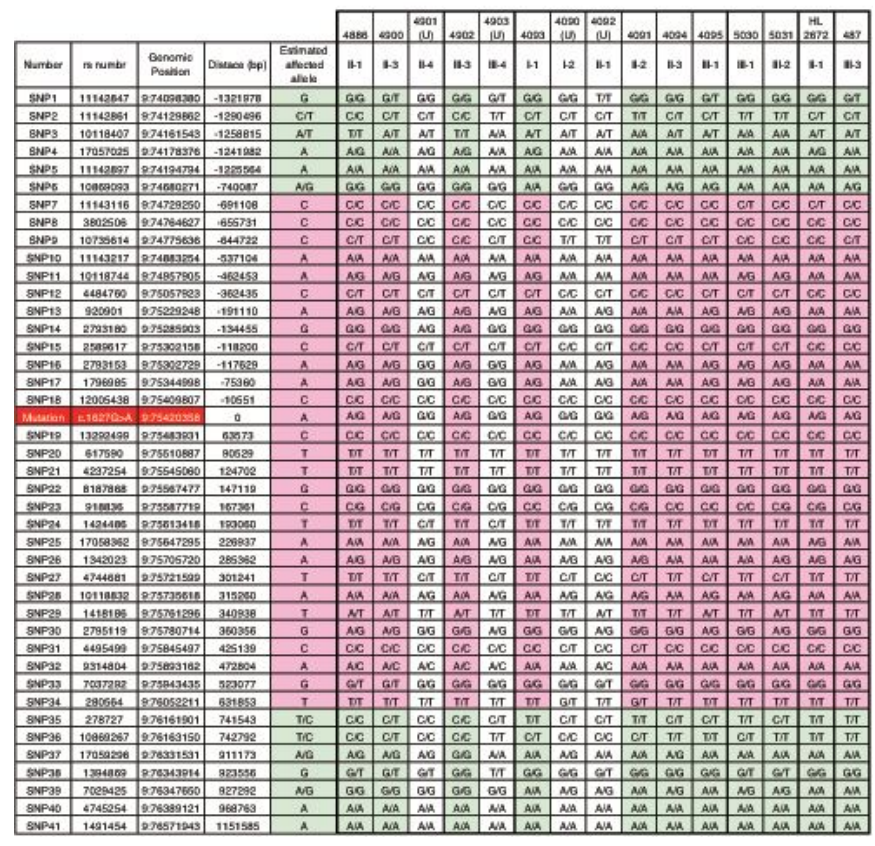

\section{Figure 2}

Haplotype analysis of the TMC1 recurrent variant c.1627G>A:p.D543N. The estimated haplotypes surrounding the 3Mbp region of this variant are indicated. The pink area was conserved between unrelated families. The green area was not conserved.

\section{Supplementary Files}

This is a list of supplementary files associated with this preprint. Click to download. 
- FigureS1.pdf

Page 9/9 